\title{
Associations of dietary glycaemic index and glycaemic load with food and nutrient intake and general and central obesity in British adults
}

\author{
Kentaro Murakami ${ }^{1,2,3 *}$, Tracy A. McCaffrey $^{1}$ and M. Barbara E. Livingstone ${ }^{1}$ \\ ${ }^{1}$ Northern Ireland Centre for Food and Health (NICHE), University of Ulster, Cromore Road, Coleraine BT52 1SA, UK \\ ${ }^{2}$ JSPS Postdoctoral Fellow for Research Abroad, Japan Society for the Promotion of Science, Tokyo 102-8472, Japan \\ ${ }^{3}$ Department of Social and Preventive Epidemiology, School of Public Health, University of Tokyo, Tokyo 113-0033, Japan
}

(Submitted 24 August 2012 - Final revision received 27 March 2013 - Accepted 8 April 2013 - First published online 9 May 2013)

\section{Abstract}

Inconsistent associations between dietary glycaemic index (GI) and glycaemic load (GL) and body fatness may be partly due to differences in the underlying dietary patterns or energy under-reporting. In the present study, we examined the cross-sectional associations of dietary GI and GL with food and nutrient intake and general and central obesity, accounting for energy under-reporting. The subjects were 1487 British adults aged 19-64 years. Dietary intake was assessed using a $7 \mathrm{~d}$ weighed dietary record. Breads and potatoes were the positive predictive foods for dietary GI, while fruit, other cereals and dairy products were the negative predictors. These foods were similarly identified in the analysis of only acceptable reporters (AR; ratio of reported energy intake:estimated energy requirement within 0.665-1.335) and under-reporters (UR; ratio <0.665). Dietary GL was closely correlated with carbohydrate intake. Multiple linear regression analyses showed that dietary GI was independently associated with a higher risk of general obesity (BMI $\geq 30 \mathrm{~kg} / \mathrm{m}^{2}$ ) and central obesity (waist circumference $\geq 102 \mathrm{~cm}$ in men and $\geq 88 \mathrm{~cm}$ in women). Dietary GL was also associated with general (only women) and central obesity. Similarly, in the analysis of AR, the GI showed positive associations with general and central obesity, and, only in women, the GL showed positive associations with general and central obesity. Conversely, in the analysis of UR, the associations were generally weaker and many of them failed to reach statistical significance. In conclusion, we found independent positive associations of dietary GI and GL with general and central obesity in British adults.

\section{Key words: Glycaemic index: Glycaemic load: Obesity: Under-reporting}

The rapid increase in obesity worldwide has inevitably been accompanied by an increasing interest in identifying modifiable lifestyle factors of excess body fatness, including dietary glycaemic index (GI) and glycaemic load (GL). A diet with a low GI or GL, due to the slower blood glucose and insulin response following consumption, is hypothesised to stimulate increased satiety and reduce voluntary energy intake (EI) ${ }^{(1)}$, reduce fat storage by regulating fuel partitioning ${ }^{(2)}$, limit the decrease of resting energy expenditure in the fasting state ${ }^{(3)}$ and in turn prevent the accumulation of body fat ${ }^{(4)}$. However, epidemiological studies on dietary GI and GL in relation to measures of body fatness in free-living adults have provided a mixture of positive ${ }^{(5-12)}$ null $^{(7,10-16)}$ and inverse $(8,9,11,14,17,18)$ associations. These heterogeneous results may reflect cultural differences in food and nutrient intake patterns associated with dietary GI and GL. Previous studies have generally shown that dietary GL is associated with many carbohydrate-rich foods and hence strongly with carbohydrate intake, while dietary GI is associated with not only a higher intake of major carbohydrate-rich foods (e.g. breads and potatoes) but also a lower intake of other foods with low-carbohydrate and noncarbohydrate nutrients (e.g. fruit and dairy products) ${ }^{(13,14,19,20)}$ However, the strength and direction of the associations of dietary GI and GL with each of the food groups and nutrients vary considerably in different contexts of food cultures ${ }^{(13,14,19,20)}$.

Alternatively, the diversity of the associations between dietary GI and GL and measures of body fatness may be due to methodological problems in dietary assessment. For example, while most studies in this area have used FFQ, with some exceptions $^{(9,12,15)}$, these instruments have not been developed specifically to measure dietary GI and GL, possibly having little utility relative to the original concept of $\mathrm{GI}^{(21)}$. In

\footnotetext{
Abbreviations: AR, acceptable reporters; EER, estimated energy requirement; EI, energy intake; GI, glycaemic index; GL, glycaemic load; MET, metabolic equivalents; NDNS, National Diet and Nutrition Survey; TEE, total energy expenditure; UR, under-reporters; WC, waist circumference; WHR, waist:hip ratio; WHtR, waist:height ratio.
} 
addition, with some exceptions ${ }^{(11,13)}$, most studies have calculated dietary GI and GL mainly based on an earlier GI table, which contains a limited number of food items (about 750) with a mixture of GI values obtained from standard and non-standard protocols in both healthy and diabetic subjects $^{(22)}$. The application of GI based on diabetic subjects to the general (healthy) population has been questioned because of the established inconsistencies in the values obtained ${ }^{(23,24)}$. More importantly, associations between dietary GI and GL and measures of body fatness may be confounded by misreporting of dietary intake, a serious problem in all dietary surveys $^{(25-32)}$. For example, a Danish study has shown a positive association of dietary GI and GL with BMI in non-low-energy reporters (ratio of $\mathrm{EI} B \mathrm{BMR} \geq 1 \cdot 14$ ), but there was no association in low-energy reporters ${ }^{(5)}$. Furthermore, in Spanish adults, an inverse relationship between dietary GL (but not GI) and BMI has been observed in non-low-energy reporters (ratio of EI:BMR $\geq 1 \cdot 20$ ) but not in low-energy reporters ${ }^{(14)}$. However, these studies have used a fixed cut-off value to identify low-energy reporters, without taking into account the physical activity level of each subject, which may result in misclassification of up to $50 \%$ of low-energy reporters ${ }^{(33,34)}$.

The aim of the present cross-sectional study in British adults was to examine the association of dietary GI and GL with food and nutrient intake and general and central obesity, taking into account energy under-reporting. Dietary GI and GL were estimated using a $7 \mathrm{~d}$ weighed dietary record and an updated GI table including about 2500 food items ${ }^{(23)}$. Energy underreporting was assessed based on EI against individualised measure of estimated energy requirement (EER).

\section{Subjects and methods}

\section{Survey design and analytical sample}

The present cross-sectional study was based on the National Diet and Nutrition Survey (NDNS): Adults Aged 19 to 64 Years. Data from the NDNS were obtained from the UK Data Archive, University of Essex. Complete details of the rationale, design and methods of the survey have been described elsewhere $^{(35)}$. Briefly, the sample was randomly selected from 152 randomly selected postal sectors within mainland Great Britain. Eligibility was defined as being aged 19-64 years and not pregnant or breast-feeding. Only one eligible adult per private household was selected at random. Data collection was conducted during a 12-month period (July 2000 to June 2001).

The present study was conducted according to the guidelines laid down in the Declaration of Helsinki, and all procedures involving human subjects were approved by a Multi-centre Research Ethics Committee and the National Health Service Local Research Ethics Committee covering each of the postal sectors. Written informed consent was obtained from all subjects.

Of 3704 potentially eligible people identified for the study, 2251 (61\% of the eligible sample) participated in the survey. For the present analysis, we excluded 736 subjects with missing information on the variables used ( $n 468$ for anthro- pometric data; $n 527$ for dietary data; $n 56$ for social class; $n 3$ for smoking status; and $n 593$ for physical activity; some subjects had more than one missing value) and twenty-eight underweight subjects $\left(\mathrm{BMI}<18.5 \mathrm{~kg} / \mathrm{m}^{2}\right)^{(36)}$. The final analysis sample comprised 1487 adults aged 19-64 years (678 men and 809 women; $40 \%$ of the eligible sample).

\section{Anthropometric measurements}

All anthropometric measurements were performed in duplicate by trained fieldworkers, and the mean value of two measurements was used in the analysis. Height (to the nearest $0 \cdot 1 \mathrm{~cm}$ ) and weight (to the nearest $0 \cdot 1 \mathrm{~kg}$ ) were measured while the subjects were barefoot and wearing light clothes only. BMI $\left(\mathrm{kg} / \mathrm{m}^{2}\right)$ was calculated as weight $(\mathrm{kg})$ divided by height $(\mathrm{m})$ squared. Waist circumference (WC) was measured at the midpoint between the iliac crest and the lower rib, and hip circumference was measured at the point of maximum circumference over the buttocks and below the iliac crest (to the nearest $0 \cdot 1 \mathrm{~cm}$ ). The waist:hip ratio (WHR) was calculated as WC divided by hip circumference, and the waist:height ratio (WHtR) was calculated as WC divided by height. General obesity was defined as $\mathrm{BMI} \geq 30 \mathrm{~kg} / \mathrm{m}^{2(36)}$. Central obesity was defined as WC $\geq 102 \mathrm{~cm}$ in men and $\geq 88 \mathrm{~cm}$ in women $^{(36)}$. Additionally, greater WHR was defined as WHR $\geq 1.00$ in men and $\geq 0.85$ in women ${ }^{(36)}$, and greater WHtR was defined as WHtR $\geq 0 \cdot 5^{(37)}$.

\section{Dietary assessment and calculation of dietary glycaemic index and glycaemic load}

Dietary data were collected using a $7 \mathrm{~d}$ weighed dietary record. A detailed description of the procedure has been published elsewhere ${ }^{(35)}$. Briefly, each subject was supplied with a set of digital food scales and recording diaries. The subject (and the main record-keeper if appropriate) was given both written and verbal instructions by trained interviewers on how to weigh and record items in the diary, with an example of a recording diary. When weighing was not possible (e.g. eating out), the subject was asked to record as much information as possible, including the brand name of the food item, the portion size consumed and details of any leftovers. Trained interviewers, who were responsible for coding the diaries, visited the subject's household at least twice during the recording period and checked the completeness of food recording, and if necessary, additional information was added. All the collected diaries were checked by trained nutritionists, who were responsible for converting descriptions of portion sizes to weights and all aspects of the diary, including coding, recorded weights and descriptions of items consumed. Estimates of daily intake for foods, energy and selected nutrients were calculated from the records of food consumption based on the Food Standards Agency nutrient data bank ${ }^{(38)}$, which is based on the McCance and Widdowson's composition of foods series ${ }^{(39)}$ and manufactures' data where applicable.

To calculate dietary GI and GL, GI values were assigned to individual food items ( $n$ 4612) in the dietary record, according to the following strategy developed based on previous 
studies $^{(24,40,41)}$. GI values were obtained from the latest international table of $\mathrm{GI}^{(23)}$. Glucose was used as the reference (GI for glucose 100).

Step 1. Determine whether the item has $<0.5 \mathrm{~g}$ carbohydrate (sum of sugars and starch) per $100 \mathrm{~g}$. If yes, assign a GI value of $0(n 707 ; 15 \cdot 3 \%)$. If no, go to step 2 .

Step 2. Determine whether there is a direct link to a food in the international GI table. If yes, assign that value ( $n 736$; $16.0 \%$ ). If no, go to step 3 .

Step 3. Determine whether there is a closely related food (based on macronutrient and fibre contents) in the international GI table. If yes, assign that GI value ( $n$ 2569; $55 \cdot 7 \%$ ). If no, go to step 4.

Step 4. Determine whether the median GI value of the food subgroup is available. If yes, assign the median GI value of the subgroup ( $n 81 ; 1.8 \%$ ). If no, go to step 5 .

Step 5. Determine whether the item is categorised to one of the following: vegetables; dairy products; sauce; dressing; alcoholic beverages; flour. If yes, assign the following nominal GI values ${ }^{(23,40)}$ : 40 for vegetables; 30 for dairy products; 60 for sauce; 30 for dressing; 65 (GI of sucrose) for alcoholic beverages; the GI value of bread made from the same flour for flour ( $n$ 449; 9.7\%). If no, go to step 6.

Step 6. Determine whether the item is categorised to one of the following: fats; egg; fish; meat; tea; coffee; spice; sugarfree foods or beverages. If yes, assign the nominal value of $0(n 62 ; 1.3 \%)$. If no, go to step 7 .

Step 7. Assign a nominal GI value of $50(n 8 ; 0 \cdot 2 \%)$.

Where possible, foods were given GI values that were derived from groups of eight or more healthy subjects with an appropriate methodology (i.e. Table A1 in the international GI table) ( $n$ 4582; 99.3\%). If the only relevant value was available from studies in subjects with diabetes or impaired glucose metabolism, or from studies using too few subjects, or showing wide variability (i.e. Table A2 in the international GI table), this value was used. If more than one eligible GI value was available for a given food, we assigned the mean of the GI values.

Dietary GL was calculated by multiplying the GI value of each individual food item by the amount $(\mathrm{g})$ of carbohydrate consumed from that food item, and then summing the products divided by 100. Dietary GI was calculated by dividing dietary GL by the total amount (g) of carbohydrate consumed, and then multiplying this value by 100 . For all dietary variables, mean daily values over $7 \mathrm{~d}$ were used in the analysis.

\section{Assessment of non-dietary variables}

The socio-economic status of each respondent (i.e. occupational social class) was self-reported and categorised as manual (i.e. skilled manual, partly skilled and unskilled occupations: social classes III (manual), IV and V) or nonmanual (i.e. professional, managerial, technical and skilled non-manual occupations: social classes I, II and III (nonmanual)). Smoking status (never, former or current) was also self-reported.

A $7 \mathrm{~d}$ physical activity diary was used concurrently with the dietary record. A detailed description of the procedure has been published elsewhere ${ }^{(35)}$. Briefly, the subjects were shown by trained interviewers as to how to record the information and were asked to record, to the nearest $10 \mathrm{~min}$, how long they spent performing various activities on that day. Trained interviewers checked the completeness of records at least twice during the recording period, and if necessary, additional information was added. Subsequently, time spent daily on sleep and light-, moderate- and vigorous-intensity activities was computed for each day of recording. Each type of activity was assigned a metabolic equivalent (MET) value from a published table: 1.0 for sleep; 2.0 for light-; 3.5 for moderate-; 7.5 for vigorous-intensity activities $^{(42)}$. The number of hours spent per $\mathrm{d}$ on each activity was multiplied by the MET value of that activity, and all MET-h products were summed to produce a total MET-h score for the day. A mean daily value over $7 \mathrm{~d}$ was used in the analysis.

\section{Evaluation of energy intake reporting}

We calculated each subject's EER, based on the information on age, weight, height and physical activity, with the use of equations published from the US Dietary Reference Intakes ${ }^{(43)}$ The equations were developed from a meta-analysis of methodologically sound studies using doubly labelled water as the criterion measure of total energy expenditure (TEE) ${ }^{(43)}$. Based on the physical activity level value, which was calculated as total MET-h/d (from the $7 \mathrm{~d}$ physical activity diary) divided by $24 \mathrm{~h}$, physical activity category was determined for each subject. The sex- and age-specific equations for use in populations with a range of weight statuses ${ }^{(43)}$ were used.

The subjects were identified as acceptable reporters (AR), under-reporters (UR) or over-reporters of EI based on their ratio of EI:EER, according to whether the individual's ratio was within, below or above the $95 \%$ confidence limits of the expected ratio of $1 \cdot 0$. The $95 \%$ confidence limits $( \pm 2$ SD cut-offs) were calculated according to the following equation:

$$
95 \% \text { Confidence limits }= \pm 2 \times \sqrt{ }\left(\mathrm{CV}_{\mathrm{EI}}^{2} / d+\mathrm{CV}_{\mathrm{EER}}^{2}+\mathrm{CV}_{\mathrm{TEE}}^{2}\right) \text {, }
$$

where $\mathrm{CV}_{\mathrm{EI}}$ is the within-person $\mathrm{CV}$ in the reported $\mathrm{EI}, d$ is the number of days of dietary assessment, $\mathrm{CV}_{\mathrm{EER}}$ is the error in the EER equations and $\mathrm{CV}_{\mathrm{TEE}}$ is the day-to-day variation in $\mathrm{TEE}^{(44)}$ The values used were $22 \cdot 8-32 \cdot 8 \%$ for $\mathrm{CV}_{\mathrm{EI}}$ (calculated from the present NDNS data; values varied depending on the sex-age-BMI stratum), 7 for $d, 7 \cdot 9-11.5 \%$ for $\mathrm{CV}_{\mathrm{EER}}$ (based on the US Dietary Reference Intakes data ${ }^{(43,44)}$; values varied depending on the sex-age-BMI stratum) and $8.2 \%$ for $\mathrm{CV}_{\text {TEE }}$ (as reported previously from doubly labelled water studies $\left.{ }^{(45)}\right)$. As the $95 \%$ confidence limits were found to be similar across the sex-age-BMI strata (32.1-36.3\%), an average of $33.5 \%$ was used in the present analysis. Thus, AR were defined as having the EI:EER ratio in the range of 0.665-1.335, UR as EI:EER $<0.665$ and over-reporters as EI:EER $>1.335$.

\section{Statistical analyses}

All statistical analyses were performed for men and women separately, using SAS statistical software (version 9.2, SAS Institute, Inc.). Differences between AR and UR (but not for 
over-reporters because of there being only a few of them) were tested by the independent-samples $t$ test (for continuous variables) and by the $\chi^{2}$ test (for categorical variables). Stepwise regression analyses were carried out to investigate the contribution of the selected nineteen food groups to the inter-individual variation in dietary GI and GL. For those food groups contributing at least $1 \%$ variation, multiple regression analyses were performed with only those predictive food groups plus EI as explanatory variables and dietary GI or GL as the response variable. The associations of intakes of energy and selected nutrients with dietary GI and GL were investigated by Spearman's correlation analyses.

Multiple linear regression analyses were performed to explore the associations of dietary GI and GL (independent variables) with measures of body fatness (dependent variables). With the use of the PROC REG procedure, we calculated the adjusted regression coefficients (with SE) of variation of BMI, WC, WHR and WHtR by a 10-unit GI increase and a 50-unit GL increase. Furthermore, multiple logistic regression analyses were performed to explore the associations of dietary GI and GL with general and central obesity in addition to greater WHR and WHtR. Using the PROC LOGISTIC procedure, adjusted OR (and 95\% CI) for these statuses per 10-unit GI increase and 50-unit GL increase were calculated. Potential confounding factors included in the multivariate models were age, social class, smoking status, physical activity, total fat intake, alcohol intake, dietary fibre intake and EI:EER. The analyses were conducted not only for the entire population but also for only AR or only UR.

The values of nutrient intake were energy adjusted using the density method (i.e. percentage of energy for energy-providing nutrients and amount per $10 \mathrm{MJ}$ of energy for dietary fibre). We used crude values for dietary GI and energy-adjusted values in the density method (per 10 MJ) for dietary GL because dietary GI is by definition a measure of carbohydrate quality, not of quantity, whereas dietary GL is a measure of the combination of carbohydrate quality and quantity ${ }^{(6,7)}$. Use of energy-adjusted values (including dietary GI) by the residual method ${ }^{(46)}$ did not change the results materially (data not shown).

Data were not weighted to take into account known sociodemographic differences between responders and non-responders, not only because the impact of this adjustment, applied as a weighting factor, for nutritional variables was extremely small ${ }^{(35)}$ but also because we were only interested in the relationships between variables, rather than in the estimates of prevalence. All reported $P$ values are two tailed, and $P$ values $<0.05$ were considered statistically significant.

\section{Results}

Under-reporting of EI compared with that of EER was, on average, $27 \%$ in men and $31 \%$ in women (Table 1). The prevalence of general and central obesity was 24 and $29 \%$ in men and 22 and $28 \%$ in women, respectively. The mean values of dietary GI and GL were 60 (SD 3) and 169 (SD 28) per $10 \mathrm{MJ}$ in men and 58 (SD 4) and 172 (SD 29) per $10 \mathrm{MJ}$ in women, respectively.
The percentages of AR and UR were 63 and $37 \%$ in men and 55 and $45 \%$ in women, respectively (only three men (0.4\%) were classified as over-reporters). In both men and women, compared with AR, UR had a lower mean value of EI and age and a higher mean value of EER, physical activity and all measures of body fatness (except WHR), and were more likely to be employed in manual occupations, be current smokers and be obese. In only women, UR had a lower mean intake of total fat and a higher mean intake of dietary fibre. In terms of dietary GI and GL, there were no differences between UR and AR in both sexes. In both men and women, UR had lower mean intakes of all food groups examined $(\mathrm{g} / \mathrm{d})$ compared with AR, except for no differences in soft drinks (both sexes), nuts and seeds (only men), and fish and fish dishes (only women) (data not shown).

In both men and women, breads and potatoes were the positive predictive food groups for dietary GI, while fruit, other cereals and dairy products (and fruit juice in only men) were the negative predictors (Table 2). In total, these food groups accounted for 38\% (men) and 34\% (women) of the variation in dietary GI. For dietary GL, $87 \%$ (men) and $83 \%$ (women) of the variation was explained by ten carbohydrate-rich food groups, all of which showed positive associations. Food groups identified as the predictors of dietary GI and GL as well as the variation explained were relatively similar in the analysis of only AR or only UR for both men and women, although the ranking of the most predictive food groups somewhat differed in some cases. For example, in men, the most contributing food group of GI was breads in AR but potatoes in UR; in women, the most contributing food group of GL was sugar, preserves and confectionery in AR but breakfast cereals in UR.

There was no association between dietary GI and EI in men, while a weak inverse association was found in women (Table 3). Dietary GI was positively associated with starch and dietary GL (and alcohol in only men) and inversely associated with SFA, total sugars and dietary fibre (and total fat in only men). Dietary GI was not associated with total carbohydrate. There was a weak inverse association between dietary GL and EI in only men. Dietary GL was positively associated with total carbohydrate, total sugars, starch and dietary fibre and inversely associated with protein, total fat, SFA and alcohol. Relatively similar correlation coefficient values were obtained when AR and UR were analysed separately.

While dietary GI showed no association with BMI in both men and women, dietary GI was independently positively associated with all the other measures of body fatness (WC, WHR and WHtR), except with WHR in women (Table 4). Dietary GL was not associated with any measure of body fatness in men. Conversely, in women, although there was no association between dietary GL and BMI, dietary GL showed an independent positive association with all the other measures of body fatness. Similarly, in the analysis including AR, dietary GI showed positive associations with all the measures of body fatness except with BMI in both sexes. Additionally, dietary GL similarly showed no associations in men, but positive associations with all the measures 
Table 1. Characteristics of the subjects*

(Mean values and standard deviations or percentages)

\begin{tabular}{|c|c|c|c|c|c|c|c|c|c|c|c|c|c|c|}
\hline & \multicolumn{6}{|c|}{ Men } & \multirow[b]{3}{*}{$P \neq$} & \multicolumn{6}{|c|}{ Women } & \multirow[b]{3}{*}{$P \neq$} \\
\hline & \multicolumn{2}{|c|}{ All $(n 678) \dagger$} & \multicolumn{2}{|c|}{$\operatorname{AR}(n 426)$} & \multicolumn{2}{|c|}{ UR ( $n$ 249) } & & \multicolumn{2}{|c|}{ All $(n 809)$} & \multicolumn{2}{|c|}{ AR ( $n$ 447) } & \multicolumn{2}{|c|}{ UR ( $n$ 362) } & \\
\hline & Mean & SD & Mean & SD & Mean & SD & & Mean & SD & Mean & SD & Mean & SD & \\
\hline El:EER & 0.73 & 0.19 & 0.83 & 0.13 & 0.54 & $0 \cdot 10$ & $<0.0001$ & 0.69 & 0.18 & 0.82 & 0.11 & 0.53 & 0.11 & $<0.0001$ \\
\hline EI (kJ/d) & 9837 & 2523 & 11038 & 1937 & 7666 & 1621 & $<0.0001$ & 6932 & 1769 & 8073 & 1258 & 5523 & 1202 & $<0.0001$ \\
\hline $\operatorname{EER}(\mathrm{kJ} / \mathrm{d})$ & 13582 & 1698 & 13263 & 1533 & 14141 & 1824 & $<0.0001$ & 10191 & 1207 & 9876 & 935 & 10582 & 1379 & $<0.0001$ \\
\hline Age (years) & $42 \cdot 4$ & $12 \cdot 0$ & 43.5 & 11.7 & $40 \cdot 6$ & $12 \cdot 2$ & 0.002 & $42 \cdot 4$ & 11.9 & 43.8 & $12 \cdot 3$ & $40 \cdot 6$ & $11 \cdot 3$ & 0.0002 \\
\hline Social class (\%) & & & & & & & 0.02 & & & & & & & 0.005 \\
\hline Manual & \multicolumn{2}{|c|}{$46 \cdot 2$} & \multicolumn{2}{|c|}{42.5} & \multicolumn{2}{|c|}{$51 \cdot 8$} & & \multicolumn{2}{|c|}{$32 \cdot 4$} & \multicolumn{2}{|c|}{28.2} & \multicolumn{2}{|c|}{$37 \cdot 6$} & \\
\hline Non-manual & \multicolumn{2}{|c|}{$53 \cdot 8$} & \multirow{2}{*}{\multicolumn{2}{|c|}{$57 \cdot 5$}} & \multicolumn{2}{|c|}{$48 \cdot 2$} & & \multicolumn{2}{|c|}{$67 \cdot 6$} & \multicolumn{2}{|c|}{$71 \cdot 8$} & \multirow{2}{*}{\multicolumn{2}{|c|}{$62 \cdot 4$}} & \\
\hline Smoking status (\%) & & & & & & & 0.06 & & & & & & & 0.02 \\
\hline Never & \multicolumn{2}{|c|}{$44 \cdot 3$} & \multirow{2}{*}{\multicolumn{2}{|c|}{$\begin{array}{l}47.2 \\
25.6\end{array}$}} & \multicolumn{2}{|c|}{39.4} & & \multicolumn{2}{|c|}{$46 \cdot 7$} & \multicolumn{2}{|c|}{$51 \cdot 0$} & \multicolumn{2}{|c|}{$41 \cdot 4$} & \\
\hline Former & \multirow{2}{*}{\multicolumn{2}{|c|}{$\begin{array}{l}25.4 \\
30.4\end{array}$}} & & & & & & & & & & & & \\
\hline Current & & & & & & & & & & & & & & \\
\hline Physical activity (MET-h/d) & $46 \cdot 1$ & $10 \cdot 2$ & $45 \cdot 4$ & 9.5 & $47 \cdot 1$ & $10 \cdot 7$ & 0.03 & $42 \cdot 3$ & $4 \cdot 1$ & $42 \cdot 0$ & 3.8 & $42 \cdot 7$ & 4.4 & 0.01 \\
\hline BMI $\left(\mathrm{kg} / \mathrm{m}^{2}\right)$ & $27 \cdot 3$ & 4.4 & $26 \cdot 6$ & 3.7 & 28.5 & $5 \cdot 0$ & $<0.0001$ & $26 \cdot 8$ & $5 \cdot 6$ & $25 \cdot 6$ & 4.7 & $28 \cdot 2$ & $6 \cdot 2$ & $<0.0001$ \\
\hline WC $(\mathrm{cm})$ & $96 \cdot 0$ & $11 \cdot 0$ & $94 \cdot 7$ & $10 \cdot 3$ & $98 \cdot 2$ & 11.9 & $<0.0001$ & 83.1 & 11.9 & $81 \cdot 2$ & $10 \cdot 9$ & 85.4 & $12 \cdot 8$ & $<0.0001$ \\
\hline WHR & 0.91 & 0.06 & 0.91 & 0.06 & 0.91 & 0.06 & 0.21 & 0.79 & 0.06 & 0.79 & 0.06 & 0.80 & 0.06 & 0.07 \\
\hline WHtR & 0.55 & 0.07 & 0.54 & 0.06 & 0.56 & 0.07 & $<0.0001$ & 0.52 & 0.08 & 0.50 & 0.07 & 0.53 & 0.08 & $<0.0001$ \\
\hline General obesity $(\%) \S$ & & & & & & & $<0.0001$ & & & & & & & $<0.0001$ \\
\hline Central obesity (\%)\| & & & & & & & $<0.0001$ & & & & & & & $<0.0001$ \\
\hline Total fat intake ( $\%$ of energy) & 33.4 & $5 \cdot 9$ & 33.6 & $5 \cdot 7$ & $33 \cdot 1$ & $6 \cdot 2$ & 0.33 & 33.6 & $6 \cdot 6$ & $34 \cdot 1$ & $6 \cdot 1$ & $33 \cdot 1$ & $7 \cdot 1$ & 0.02 \\
\hline Alcohol intake (\% of energy) & $6 \cdot 7$ & $7 \cdot 3$ & $7 \cdot 0$ & $7 \cdot 1$ & $6 \cdot 1$ & $7 \cdot 7$ & 0.13 & $4 \cdot 0$ & 5.5 & $4 \cdot 2$ & 5.5 & $3 \cdot 8$ & $5 \cdot 6$ & 0.25 \\
\hline Dietary fibre intake (g/10 MJ) & $16 \cdot 0$ & 5.5 & $15 \cdot 9$ & $5 \cdot 1$ & $16 \cdot 2$ & $6 \cdot 1$ & 0.58 & $18 \cdot 7$ & $7 \cdot 1$ & $18 \cdot 0$ & $6 \cdot 2$ & $19 \cdot 6$ & 8.0 & 0.002 \\
\hline Dietary GIף & $59 \cdot 5$ & $3 \cdot 4$ & $59 \cdot 4$ & $3 \cdot 1$ & $59 \cdot 6$ & 3.9 & $0 \cdot 70$ & $58 \cdot 3$ & $3 \cdot 7$ & $58 \cdot 3$ & 3.4 & $58 \cdot 4$ & $4 \cdot 1$ & 0.67 \\
\hline Dietary GL (per 10 MJ) q & 169 & 28 & 168 & 26 & 169 & 31 & 0.52 & 172 & 29 & 173 & 26 & 171 & 31 & 0.19 \\
\hline
\end{tabular}

AR, acceptable reporters; UR, under-reporters; El:EER, ratio of energy intake:estimated energy requirement; El, energy intake; EER, estimated energy requirement; MET, metabolic equivalents; WC, waist circumference; WHR, waist:hip ratio; WHtR, waist:height ratio; Gl, glycaemic index; GL, glycaemic load.

*AR were defined as subjects with El:EER 0.665-1.335; UR were defined as subjects with El:EER $<0.665$.

t Including over-reporters of EI $(n 3)$, defined as subjects with EI:EER $>1.335$

$\ddagger P$ values for differences between AR and UR based on the independent-samples $t$ test for continuous variables and the $\chi^{2}$ test for categorical variables.

$\S \mathrm{BMI} \geq 30 \mathrm{~kg} / \mathrm{m}^{2}$.

UWC $>102 \mathrm{~cm}$ for men and $\geq 88 \mathrm{~cm}$ for women.

I Based on the Gl of glucose (100). 
Table 2. Food groups contributing to the inter-individual variation in dietary glycaemic index (GI) and glycaemic load (GL)*

(Regression coefficients with their standard errors and partial determination coefficients)

\begin{tabular}{|c|c|c|c|c|c|c|c|c|c|}
\hline \multirow[b]{2}{*}{ Food groups† } & \multicolumn{3}{|c|}{ All } & \multicolumn{3}{|c|}{$A R$} & \multicolumn{3}{|c|}{ UR } \\
\hline & $\beta \ddagger$ & SE‡ & Partial $R^{2}$ & $\beta \ddagger$ & SE‡ & Partial $R^{2}$ & $\beta \ddagger$ & SE‡ & Partial $R^{2}$ \\
\hline Men & \multicolumn{3}{|c|}{$n 678 \S$} & \multicolumn{3}{|c|}{$n 426$} & \multicolumn{3}{|c|}{$n 249$} \\
\hline Dietary GI & \multicolumn{3}{|c|}{ Model $R^{2} 0.38$} & \multicolumn{3}{|c|}{ Model $R^{2} 0.41$} & \multicolumn{3}{|c|}{ Model $R^{2} 0.42$} \\
\hline Breads & $2 \cdot 19$ & 0.19 & 0.12 & $2 \cdot 32$ & 0.21 & 0.17 & $2 \cdot 50$ & 0.37 & 0.10 \\
\hline Fruit & -0.56 & $0 \cdot 10$ & 0.09 & -0.38 & 0.10 & 0.07 & -1.09 & 0.25 & 0.08 \\
\hline Potatoes & $1 \cdot 21$ & 0.17 & 0.07 & 1.06 & 0.18 & 0.03 & 1.92 & 0.35 & 0.17 \\
\hline Other cereals & -0.70 & 0.14 & 0.04 & -0.50 & 0.15 & 0.09 & -0.73 & 0.27 & 0.03 \\
\hline Dairy products & -0.21 & 0.06 & 0.02 & -0.13 & 0.06 & 0.02 & -0.35 & 0.14 & 0.01 \\
\hline Fruit juice & -0.50 & 0.11 & 0.02 & -0.40 & 0.12 & 0.02 & -0.82 & 0.24 & 0.02 \\
\hline Breakfast cereals & $-\|$ & $-\|$ & $-\|$ & $-\|$ & $-\|$ & $-\|$ & 1.60 & 0.63 & 0.01 \\
\hline Dietary GL & \multicolumn{3}{|c|}{ Model $R^{2} 0.87$} & \multicolumn{3}{|c|}{ Model $R^{2} 0.82$} & \multicolumn{3}{|c|}{ Model $R^{2} 0.84$} \\
\hline Breads & $28 \cdot 49$ & 1.35 & 0.28 & $30 \cdot 23$ & $1 \cdot 17$ & 0.21 & $27 \cdot 68$ & 2.06 & 0.19 \\
\hline Sugar, preserves and confectionery & $42 \cdot 80$ & $2 \cdot 22$ & $0 \cdot 20$ & $44 \cdot 60$ & $2 \cdot 8$ & 0.17 & $46 \cdot 22$ & 3.65 & 0.15 \\
\hline Biscuits, cakes and pastries & $22 \cdot 75$ & 1.88 & 0.12 & $22 \cdot 22$ & $2 \cdot 24$ & 0.12 & $24 \cdot 27$ & 3.50 & 0.06 \\
\hline Breakfast cereals & 21.93 & 1.66 & 0.07 & $20 \cdot 41$ & $2 \cdot 03$ & 0.08 & $24 \cdot 34$ & 2.90 & 0.10 \\
\hline Potatoes & $13 \cdot 66$ & $1 \cdot 15$ & 0.05 & $13 \cdot 07$ & 1.46 & 0.03 & $15 \cdot 99$ & 1.68 & 0.11 \\
\hline Soft drinks, not low energy & 3.68 & 0.34 & 0.05 & $3 \cdot 70$ & 0.44 & 0.06 & 3.93 & 0.48 & 0.09 \\
\hline Other cereals & $10 \cdot 73$ & 0.93 & 0.03 & $9 \cdot 30$ & $1 \cdot 20$ & 0.05 & $13 \cdot 03$ & 1.31 & 0.06 \\
\hline Alcoholic beverages & 0.49 & $0 \cdot 15$ & 0.03 & 0.61 & 0.19 & 0.02 & 0.67 & 0.23 & 0.03 \\
\hline Dairy products & $2 \cdot 71$ & 0.40 & 0.02 & 2.59 & 0.51 & 0.02 & 2.92 & 0.70 & 0.02 \\
\hline Fruit juice & 5.03 & 0.72 & 0.02 & 4.95 & 0.88 & 0.02 & $5 \cdot 39$ & $1 \cdot 20$ & 0.02 \\
\hline Meat and meat products & $-\|$ & $-\|$ & $-\|$ & $-\|$ & $-\|$ & $-\|$ & $1 \cdot 31$ & $1 \cdot 19$ & 0.01 \\
\hline Fruit & $-\|$ & $-\|$ & $-\|$ & 4.49 & 0.79 & 0.03 & $-\|$ & $-\|$ & $-\|$ \\
\hline Women & \multicolumn{3}{|c|}{$n 809$} & \multicolumn{3}{|c|}{$n 447$} & \multicolumn{3}{|c|}{$n 362$} \\
\hline Dietary GI & \multicolumn{3}{|c|}{ Model $R^{2} 0.34$} & \multicolumn{3}{|c|}{ Model $R^{2} 0.36$} & \multicolumn{3}{|c|}{ Model $R^{2} 0.38$} \\
\hline Potatoes & 2.56 & $0 \cdot 21$ & 0.16 & 1.94 & 0.24 & 0.16 & 3.73 & 0.36 & 0.18 \\
\hline Fruit & -0.66 & 0.10 & 0.06 & -0.59 & 0.11 & 0.05 & -0.93 & 0.17 & 0.07 \\
\hline Breads & $2 \cdot 70$ & 0.28 & 0.05 & $2 \cdot 22$ & 0.32 & 0.08 & 4.01 & 0.52 & 0.06 \\
\hline Other cereals & -0.89 & 0.19 & 0.04 & -1.09 & $0 \cdot 22$ & 0.05 & -0.57 & 0.32 & 0.02 \\
\hline Dairy products & -0.31 & 0.07 & 0.03 & -0.26 & 0.08 & 0.02 & -0.53 & 0.13 & 0.03 \\
\hline Breakfast cereals & $-\|$ & $-\|$ & $-\|$ & $-\|$ & $-\|$ & $-\|$ & 2.59 & 0.62 & 0.02 \\
\hline Dietary GL & \multicolumn{3}{|c|}{ Model $R^{2} 0.83$} & \multicolumn{3}{|c|}{ Model $R^{2} 0.71$} & \multicolumn{3}{|c|}{ Model $R^{2} 0.77$} \\
\hline Sugar, preserves and confectionery & $36 \cdot 94$ & $2 \cdot 02$ & 0.21 & $35 \cdot 81$ & 2.59 & 0.21 & $39 \cdot 01$ & 3.53 & 0.11 \\
\hline Breads & $23 \cdot 29$ & 1.33 & 0.17 & $23 \cdot 26$ & 1.82 & 0.11 & 23.09 & $2 \cdot 00$ & 0.14 \\
\hline Biscuits, cakes and pastries & $17 \cdot 39$ & 1.85 & 0.10 & $17 \cdot 04$ & $2 \cdot 30$ & 0.06 & $16 \cdot 01$ & 3.65 & 0.04 \\
\hline Breakfast cereals & $17 \cdot 16$ & 1.38 & 0.09 & $15 \cdot 28$ & $1 \cdot 78$ & 0.07 & $22 \cdot 55$ & $2 \cdot 32$ & 0.18 \\
\hline Potatoes & $14 \cdot 38$ & 0.99 & 0.07 & 12.99 & $1 \cdot 38$ & 0.05 & $16 \cdot 73$ & 1.39 & 0.11 \\
\hline Other cereals & $9 \cdot 35$ & 0.88 & 0.07 & $8 \cdot 42$ & $1 \cdot 25$ & 0.07 & $10 \cdot 54$ & $1 \cdot 24$ & 0.11 \\
\hline Soft drinks, not low energy & $4 \cdot 13$ & 0.32 & 0.05 & $4 \cdot 20$ & 0.41 & 0.07 & 3.65 & 0.54 & 0.03 \\
\hline Fruit & 3.49 & 0.46 & 0.03 & $3 \cdot 33$ & 0.63 & 0.03 & $3 \cdot 71$ & 0.65 & 0.03 \\
\hline Dairy products & 2.08 & 0.34 & 0.02 & 2.03 & 0.47 & 0.02 & 1.95 & 0.50 & 0.02 \\
\hline Fruit juice & 3.03 & 0.62 & 0.01 & 2.99 & 0.82 & 0.02 & $-\|$ & $-\|$ & $-\|$ \\
\hline
\end{tabular}

AR, acceptable reporters; UR, under-reporters; $\beta$, Regression coefficient.

${ }^{*} A R$ were defined as subjects with the ratio of energy intake:estimated energy requirement (El:EER) $0.665-1.335$; UR were defined as subjects with El:EER $<0.665$

†Food groups listed are those contributing at least $1 \%$ of the variation of dietary GI or GL based on the stepwise regression analysis with nineteen food groups (i.e. breads; breakfast cereals; biscuits, cakes and pastries; other cereals; dairy products; egg and egg dishes; butter and spreads; meat and meat products; fish and fish dishes; vegetables; potatoes; fruit; sugar, preserves and confectionery; fruit juice; alcoholic beverages; tea, coffee and water; nuts and seeds; soft drinks, not low energy; and soft drinks, low energy) as explanatory variables and dietary GI or GL as the response variable.

$\ddagger$ Models with the listed variables and energy intake as the explanatory variables and dietary GI or GL as the response variable; regression coefficients mean the change of dietary GI or GL with a $100 \mathrm{~g}$ increase of each food group.

$\S$ Including over-reporters of energy intake $(n 3)$, defined as subjects with El:EER $>1.335$.

$\|$ Not contributing at least $1 \%$ of the variation of dietary GI or GL.

of body fatness except with BMI in women. Conversely, in the analysis of UR, the associations were generally weaker, with significant associations only being observed between GI and WHR in men and GL and WC and WHtR in women.

Table 5 presents the independent association between dietary GI and GL and general and central obesity. Dietary GI was associated with a higher risk of both general and central obesity in both men $(P=0.009$ and 0.004, respectively) and women $(P=0.02$ and 0.0007 , respectively). Dietary GL was associated with a higher risk of general obesity in only women $(P=0.03)$ and central obesity in both men and women $(P=0.03$ and $0 \cdot 0008$, respectively). Similarly, in the analysis of AR, dietary GI showed positive associations with both general and central obesity in both men ( $P=0.052$ and 0.02 , respectively) and women $(P=0.04$ and 0.002 , respectively). Dietary GL also showed positive associations with both general and central obesity in women ( $P=0.04$ and 0.004 , respectively), although there was no association with central obesity in men. Conversely, in the analysis of UR, although the direction of the associations was similar, the associations were generally 
Table 3. Correlation of energy and nutrient intake with dietary glycaemic index (GI) and glycaemic load (GL)†

(Spearman's correlation coefficients)

\begin{tabular}{|c|c|c|c|c|c|c|}
\hline & \multicolumn{2}{|c|}{ All } & \multicolumn{2}{|c|}{ AR } & \multicolumn{2}{|c|}{ UR } \\
\hline & $\mathrm{Gl}$ & $\mathrm{GL}$ & GI & $\mathrm{GL}$ & GI & $\mathrm{GL}$ \\
\hline & $r$ & $r$ & $r$ & $r$ & $r$ & $r$ \\
\hline Men & \multicolumn{2}{|c|}{$n 678 \neq$} & \multicolumn{2}{|c|}{$n 426$} & \multicolumn{2}{|c|}{$n 249$} \\
\hline Energy & -0.04 & $-0.08^{\star}$ & -0.04 & $-0.10^{*}$ & 0.02 & -0.07 \\
\hline Protein & 0.03 & $-0.17^{\star}$ & 0.02 & $-0.16^{\star}$ & 0.02 & $-0 \cdot 19^{*}$ \\
\hline Total fat & $-0.11^{*}$ & $-0.39^{\star}$ & -0.09 & $-0.37^{\star}$ & $-0.15^{\star}$ & $-0.43^{*}$ \\
\hline SFA & $-0.13^{\star}$ & $-0.25^{\star}$ & $-0.13^{*}$ & $-0.24^{\star}$ & -0.11 & $-0.27^{\star}$ \\
\hline Total carbohydrate & -0.03 & $0.93^{*}$ & -0.06 & $0.94^{*}$ & 0.02 & $0.93^{*}$ \\
\hline Total sugars & $-0.22^{\star}$ & $0.51^{*}$ & $-0.25^{*}$ & $0.54^{*}$ & $-0.16^{\star}$ & $0.48^{*}$ \\
\hline Starch & $0.20^{*}$ & $0.57^{\star}$ & $0.20^{*}$ & $0.62^{*}$ & $0.20^{*}$ & $0.50^{*}$ \\
\hline Alcohol & $0.11^{*}$ & $-0.48^{\star}$ & 0.09 & $-0.54^{\star}$ & $0 \cdot 15^{\star}$ & $-0.41^{*}$ \\
\hline Dietary fibre & $-0.14^{\star}$ & $0.29^{\star}$ & $-0 \cdot 16^{*}$ & $0.35^{*}$ & -0.10 & $0.20^{*}$ \\
\hline Dietary GI & 1 & $0.30^{*}$ & 1 & $0.26^{\star}$ & 1 & $0.36^{*}$ \\
\hline Dietary GL & $0.30^{\star}$ & 1 & $0.26^{*}$ & 1 & $0.36^{\star}$ & 1 \\
\hline Women & \multicolumn{2}{|c|}{$n 809$} & \multicolumn{2}{|c|}{$n 447$} & \multicolumn{2}{|c|}{$n 362$} \\
\hline Energy & $-0.07^{\star}$ & -0.02 & -0.01 & -0.07 & $-0 \cdot 10^{*}$ & -0.09 \\
\hline Protein & -0.03 & $-0.14^{\star}$ & -0.07 & $-0.13^{*}$ & -0.02 & $-0 \cdot 16^{\star}$ \\
\hline Total fat & -0.05 & $-0.57^{\star}$ & -0.03 & $-0.53^{\star}$ & -0.05 & $-0.62^{*}$ \\
\hline SFA & $-0.09^{\star}$ & $-0.45^{\star}$ & -0.08 & $-0.46^{\star}$ & -0.09 & $-0.45^{\star}$ \\
\hline Total carbohydrate & 0.01 & $0.92^{*}$ & 0.04 & $0.92^{*}$ & -0.02 & $0.91^{*}$ \\
\hline Total sugars & $-0.27^{\star}$ & $0.46^{*}$ & $-0.22^{*}$ & $0.48^{\star}$ & $-0.31^{\star}$ & $0.47^{*}$ \\
\hline Starch & $0.31^{*}$ & $0.55^{\star}$ & $0.27^{\star}$ & $0.54^{*}$ & $0.33^{*}$ & $0.59^{*}$ \\
\hline Alcohol & 0.02 & $-0.39^{\star}$ & 0.04 & $-0.42^{\star}$ & -0.01 & $-0.37^{*}$ \\
\hline Dietary fibre & $-0.14^{\star}$ & $0.28^{*}$ & $-0.19^{\star}$ & $0.23^{\star}$ & -0.09 & $0.34^{*}$ \\
\hline Dietary GI & 1 & $0.37^{\star}$ & 1 & $0.40^{\star}$ & 1 & $0.34^{*}$ \\
\hline Dietary GL & $0.37^{\star}$ & 1 & $0.40^{*}$ & 1 & $0.34^{*}$ & 1 \\
\hline
\end{tabular}

AR, acceptable reporters; UR, under-reporters.

${ }^{*} P<0.05$.

†Values are Spearman's correlation coefficients calculated using energy-adjusted values (i.e. percentage of energy for energy-providing nutrients and amount per $10 \mathrm{MJ}$ of energy for dietary fibre and $\mathrm{GL}$ ) except for energy and dietary GI (for which crude values were used). Dietary GI and GL were calculated based on the GI of glucose (100). AR were defined as subjects with the ratio of energy intake:estimated energy requirement (El:EER) 0.665-1.335; UR were defined as subjects with El:EER $<0.665$.

$\ddagger$ Including over-reporters of energy intake $(n 3)$, defined as subjects with El:EER $>1.335$.

weaker and many of them failed to reach statistical significance $(P=0 \cdot 06-0 \cdot 13)$, except that between dietary GL and central obesity in women $(P=0 \cdot 03)$. Excluding EI:EER from the models did not change the results materially (data not shown). When greater WHR and WHtR were used as dependent variables, only a few significant positive associations were observed (data not shown): those between dietary GL and greater WHtR in all women $(P=0.002)$ and female AR $(P=0.03)$ and those between dietary GI and greater WHR in all men $(P=0.03)$, male $\mathrm{AR}(P=0.03)$ and female $\mathrm{AR}(P=0.04)$.

Excluding 397 subjects who reported dieting or that illness had affected their eating during the diet-recording period did not alter the findings. The exactly same food groups presented in Table 2 were identified as the predictors of dietary GI and GL in both men (model $R^{2} 0 \cdot 39$ and $0 \cdot 88$, respectively) and women (model $R^{2} 0.37$ and 0.82 , respectively), in addition to fruit for GL in men and vegetables for GI in women. Dietary GL was closely correlated with carbohydrate intake (Spearman's $r 0.92$ in both sexes). Dietary GI was associated with a higher risk of both general and central obesity in both men (adjusted OR 2·87, $95 \%$ CI 1.46, 5.66; $P=0 \cdot 002$ and adjusted OR $3.57,95 \%$ CI $1.80,7 \cdot 07 ; P=0 \cdot 003$, respectively) and women (adjusted OR 1.94, 95\% CI 0.95, 3.95;
$P=0.07$ and adjusted OR 2.59, 95\% CI $1.37,4.91 ; P=0.003$ respectively). Dietary GL was associated with a higher risk of central obesity in both men and women (adjusted OR 4.29, 95\% CI 1.76, 10.42; $P=0.001$ and adjusted OR 2.49, $95 \%$ CI $1 \cdot 18,5 \cdot 22 ; P=0 \cdot 02$, respectively).

\section{Discussion}

The present cross-sectional study in British adults showed that a high-GI diet, characterised by a high intake of breads and potatoes and a low intake of fruit, other cereals and dairy products, was independently associated with an increasing risk of general and central obesity in both men and women. Dietary GL, which was closely correlated with carbohydrate intake, was independently associated with a higher risk of central obesity in both sexes and general obesity only in women. Generally, similar associations were observed in the analysis of AR, while the associations were generally weaker and many of them did not reach statistical significance in the analysis of UR. Thus, the associations between dietary GI and GL and general and central obesity seemed to be confounded by energy under-reporting. To our knowledge, this is the first study to examine dietary GI and GL in relation to food and nutrient intake and measures of body fatness, taking into 
Table 4. Associations of dietary glycaemic index (Gl) and glycaemic load (GL) with the measures of body fatness* (Regression coefficients with their standard errors)

\begin{tabular}{|c|c|c|c|c|c|c|c|c|c|}
\hline & \multicolumn{3}{|c|}{ All } & \multicolumn{3}{|c|}{ AR } & \multicolumn{3}{|c|}{ UR } \\
\hline & $\beta \dagger$ & SE† & $P$ & $\beta \dagger$ & SE† & $P$ & $\beta \dagger$ & SE† & $P$ \\
\hline Men & \multicolumn{3}{|c|}{$n 678 \ddagger$} & \multicolumn{3}{|c|}{$n 426$} & \multicolumn{3}{|c|}{$n 249$} \\
\hline \multicolumn{10}{|c|}{ Dietary GI (10 units)§ } \\
\hline BMI $\left(\mathrm{kg} / \mathrm{m}^{2}\right)$ & 0.80 & 0.48 & 0.09 & 1.02 & 0.59 & 0.08 & 0.60 & 0.82 & 0.46 \\
\hline WC $(\mathrm{cm})$ & $3 \cdot 22$ & $1 \cdot 17$ & 0.006 & 3.09 & 1.57 & 0.049 & $3 \cdot 19$ & 1.85 & 0.09 \\
\hline WHR & 0.020 & 0.006 & 0.002 & 0.020 & 0.009 & 0.03 & 0.020 & 0.009 & 0.03 \\
\hline WHtR & 0.019 & 0.007 & 0.005 & 0.021 & 0.009 & 0.02 & 0.017 & 0.011 & 0.11 \\
\hline \multicolumn{10}{|c|}{ Dietary GL (50 units/10 MJ)§ } \\
\hline BMI $\left(\mathrm{kg} / \mathrm{m}^{2}\right)$ & -0.41 & 0.61 & 0.50 & -0.66 & 0.75 & 0.38 & -0.05 & 1.03 & 0.96 \\
\hline WC (cm) & 1.74 & 1.50 & 0.25 & 0.93 & $2 \cdot 02$ & 0.64 & $2 \cdot 77$ & $2 \cdot 33$ & 0.24 \\
\hline WHR & 0.013 & 0.008 & 0.12 & 0.013 & 0.012 & 0.28 & 0.013 & 0.011 & 0.25 \\
\hline $\mathrm{WHtR}$ & 0.006 & 0.009 & 0.46 & 0.003 & 0.012 & 0.80 & 0.012 & 0.013 & 0.39 \\
\hline Women & & $n 809$ & & & $n 447$ & & & $n 362$ & \\
\hline \multicolumn{10}{|c|}{ Dietary GI (10 units)§ } \\
\hline $\mathrm{BMI}\left(\mathrm{kg} / \mathrm{m}^{2}\right)$ & 0.81 & 0.51 & 0.12 & 0.60 & 0.66 & 0.36 & 0.90 & 0.81 & 0.26 \\
\hline WC (cm) & $2 \cdot 69$ & 1.08 & 0.01 & 2.95 & 1.48 & 0.047 & $2 \cdot 33$ & 1.61 & 0.15 \\
\hline WHR & 0.009 & 0.006 & 0.10 & 0.019 & 0.008 & 0.02 & 0.002 & 0.008 & 0.84 \\
\hline $\mathrm{WHtR}$ & 0.017 & 0.007 & 0.01 & 0.020 & 0.010 & 0.04 & 0.014 & 0.010 & 0.17 \\
\hline \multicolumn{10}{|c|}{ Dietary GL (50 units/10MJ)§ } \\
\hline BMI $\left(\mathrm{kg} / \mathrm{m}^{2}\right)$ & 1.05 & 0.63 & 0.09 & 0.81 & 0.83 & 0.33 & 1.40 & 0.97 & 0.15 \\
\hline WC $(\mathrm{cm})$ & 3.91 & 1.33 & 0.003 & 3.37 & 1.86 & 0.07 & 4.60 & 1.93 & 0.02 \\
\hline WHR & 0.016 & 0.007 & 0.02 & 0.022 & 0.010 & 0.03 & 0.012 & 0.009 & 0.20 \\
\hline WHtR & 0.026 & 0.008 & 0.002 & 0.025 & 0.012 & 0.04 & 0.029 & 0.012 & 0.02 \\
\hline
\end{tabular}

AR, acceptable reporters; UR, under-reporters; $\beta$, regression coefficient; WC, waist circumference; WHR, waist:hip ratio; WHtR, waist:height ratio.

${ }^{*}$ Adjustment was made for age (years, continuous), social class (manual or non-manual), smoking status (never, former or current), physical activity (metabolic equivalents-h/d, continuous), total fat intake (\% of energy, continuous), alcohol intake (\% of energy, continuous), dietary fibre intake ( $\mathrm{g} / 10 \mathrm{MJ}$, continuous) and ratio of energy intake:estimated energy requirement (El:EER, continuous). AR were defined as subjects with El:EER 0.6651.335; UR were defined as subjects with El:EER $<0.665$.

†Regression coefficients mean the change of body fatness measures with a 10-unit GI increase and a 50 -unit GL (per 10 MJ) increase.

$\ddagger$ Including over-reporters of energy intake $(n 3)$, defined as subjects with El:EER $>1.335$.

$\S$ Based on the Gl of glucose (100).

account EI misreporting assessed by individualised measures of EER

Misreporting of dietary intake is a common phenomenon and appears to occur non-randomly ${ }^{(25-32)}$ and to be selective for different kinds of foods and hence nutrients ${ }^{(28-31)}$. The resulting potential for differential errors in dietary data complicates the interpretation of studies on diet and health and, at worst, might produce spurious diet-health relationships ${ }^{(25,26,29)}$. In the present study, the prevalence of EI misreporters (i.e. UR and over-reporters) was $37 \%$ in men and $45 \%$ in women, which was relatively similar to that observed in previous studies using similar dietary assessment methodologies $\left(37 \%{ }^{(32)}\right.$ and $\left.38 \%{ }^{(33)}\right)$. The results are also consistent with numerous previous studies $^{(25-32,47)}$, which have shown that UR had considerably different characteristics compared with AR, including greater body fatness, younger age, lower socio-economic status and current smoking. Particularly, higher physical activity and EER were associated with EI under-reporting. This appears reasonable, given that active subjects with a greater energy requirement can fall into the category of under-reporting ${ }^{(48)}$. Alternatively, this may be due to over-reporting of physical activity. Despite these differences, the associations of dietary GI and GL with food and nutrient intake observed in UR were relatively similar to those observed in AR. It is reasonable to assume that the observed associations in UR are not artefacts produced by energy under-reporting. Thus, the exclusion of UR in the present analysis was not warranted. The exact reason for this observation is unknown, and we are unaware of previous studies where the associations among dietary variables have been compared according to the categories of dietary misreporting status. However, it may be because the calculation of dietary GI and GL is based on only carbohydrate intake, independent of fat (which seems to be more prone to misreporting than other macronutrients) ${ }^{(28-31)}$ as well as energy. In addition, the influence of energy under-reporting may be minimised by energy adjustment, as misreporting of any food and nutrient should be correlated with EI misreporting, at least to some extent ${ }^{(28,31)}$.

A limited number of studies have suggested that the influence of dietary misreporting on the association between diet and health depends on dietary variables ${ }^{(26,29,44,49)}$ or dietary assessment methods ${ }^{(28,50,51)}$. In the present study, the positive association between dietary GI and general and central obesity observed in the total male and female populations and the positive association between dietary GL and central obesity observed in the total male and female populations and general obesity in the total female population were also observed in AR. Conversely, in the analysis of UR, many of the associations failed to reach statistical significance. Thus, although the associations seemed to be confounded by energy underreporting, there was no evidence that the association between dietary GI and GL and obesity was distorted by energy under-reporting in the present study. In line with this observation, previous studies have shown that the associations of 
Table 5. Associations of dietary glycaemic index (Gl) and glycaemic load (GL) with general and central obesity* (Odds ratios and $95 \%$ confidence intervals)

\begin{tabular}{|c|c|c|c|c|c|c|c|c|c|}
\hline & \multicolumn{3}{|c|}{ All } & \multicolumn{3}{|c|}{ AR } & \multicolumn{3}{|c|}{ UR } \\
\hline & OR† & $95 \% \mathrm{Cl} \dagger$ & $P$ & OR† & $95 \% \mathrm{Cl} \dagger$ & $P$ & OR† & $95 \% \mathrm{Cl} \dagger$ & $P$ \\
\hline Men & \multicolumn{3}{|c|}{$n 678 \ddagger$} & \multicolumn{3}{|c|}{$n 426$} & \multicolumn{3}{|c|}{$n 249$} \\
\hline \multicolumn{10}{|l|}{ Dietary GI (10 units)§ } \\
\hline General obesity & $2 \cdot 12$ & $1.20,3.73$ & 0.009 & $2 \cdot 32$ & $0.99,5.44$ & 0.052 & 1.89 & $0.87,4.12$ & 0.11 \\
\hline Central obesity & $2 \cdot 30$ & $1.30,4.05$ & 0.004 & 2.69 & $1 \cdot 16,6 \cdot 20$ & 0.02 & 2.05 & $0.94,4.47$ & 0.07 \\
\hline \multicolumn{10}{|c|}{ Dietary GL (50 units/10 MJ)§ } \\
\hline General obesity & 0.98 & $0.49,1.95$ & 0.95 & 0.92 & $0.31,2.73$ & 0.88 & 1.01 & $0.40,2.56$ & 0.98 \\
\hline Central obesity & $2 \cdot 16$ & $1 \cdot 06,4.38$ & 0.03 & 2.06 & $0.70,6.04$ & $0 \cdot 19$ & $2 \cdot 20$ & $0.47,10 \cdot 36$ & 0.11 \\
\hline Women & & $n 809$ & & & $n 447$ & & & $n 362$ & \\
\hline \multicolumn{10}{|l|}{ Dietary GI (10 units)§ } \\
\hline General obesity & 1.83 & $1.11,3.02$ & 0.02 & 2.53 & $1.05,6.06$ & 0.04 & 1.68 & $0.89,3.15$ & 0.11 \\
\hline Central obesity & $2 \cdot 29$ & $1.42,3.69$ & 0.0007 & $3 \cdot 37$ & $1 \cdot 55,7 \cdot 30$ & 0.002 & 1.77 & $0.97,3.26$ & 0.06 \\
\hline \multicolumn{10}{|c|}{ Dietary GL (50 units/10 MJ)§ } \\
\hline General obesity & 1.91 & $1.05,3.48$ & 0.03 & $3 \cdot 16$ & $1 \cdot 05,9.47$ & 0.04 & 1.75 & $0.84,3.64$ & 0.13 \\
\hline Central obesity & $2 \cdot 64$ & $1.49,4.66$ & 0.0008 & $4 \cdot 13$ & $1.58,10 \cdot 76$ & 0.004 & $2 \cdot 18$ & $1.07,4.46$ & 0.03 \\
\hline
\end{tabular}

AR, acceptable reporters; UR, under-reporters.

${ }^{*}$ Adjustment was made for age (years, continuous), social class (manual or non-manual), smoking status (never, former or current), physical activity (metabolic equivalents-h/d, continuous), total fat intake (\% of energy, continuous), alcohol intake (\% of energy, continuous), dietary fibre intake (g/10 MJ, continuous) and ratio of energy intake:estimated energy requirement (EI:EER, continuous). AR were defined as subjects with EI:EER 0.665-1.335; UR were defined as subjects with EI:EER $<0.665$.

† OR for general and central obesity per 10-unit increase of Gl and 50-unit increase of GL (per 10 MJ).

¥Including over-reporters of energy intake $(n 3)$, defined as subjects with El:EER $>1.335$.

$\S$ Based on the Gl of glucose (100).

carbohydrate intake (expressed as percentage of energy) with $\mathrm{BMI}^{(44,49)}$ and fasting plasma insulin ${ }^{(29)}$ were not affected by EI misreporting.

While we found the positive association of dietary GI with general and central obesity in both men and women, dietary GL was associated with central obesity in both sexes but with general obesity only in women. As dietary GL was strongly correlated with carbohydrate intake, as has been shown in several previous studies ${ }^{(11,13)}$, dietary GL might have provided little information beyond the carbohydrate content of the diet compared with dietary $\mathrm{GI}^{(11,21)}$. Furthermore, central obesity may be more susceptible to dietary GI and GL than general obesity given that visceral fat is more vulnerable to the influence of high insulin responses stimulated by high-GI foods compared with subcutaneous fat ${ }^{(11)}$. This may explain why we did find significant associations of dietary GI and GL with measures of central fatness (WC, WHR and WHtR), but not with a measure of general fatness (BMI) as well as somewhat stronger associations with central obesity than with general obesity. Men may also be less susceptible than women to the proposed adverse effects of high dietary GI and GL on body fatness ${ }^{(12)}$, which may account for the generally weaker associations observed in men than in women. It is not clear why we did find the association for central obesity (defined by WC) but not for greater WHR or WHtR in this population. The WC cut-offs may be more sensitive than the WHR and WHtR cut-offs, at least in this population, as the associations were similar when WC, WHR and WHtR were treated as continuous variables.

The advantages of the present study include the use of a $7 \mathrm{~d}$ weighed dietary record, a systematic assignment of GI values based on an updated and more representative GI table, measured anthropometric data and the use of individualised measure of EER to identify EI misreporters. However, there are also several limitations. First, the cross-sectional nature of the study does not permit the assessment of causality, owing to the uncertain temporality of the association. Only a prospective study taking into account dietary misreporting would provide a better understanding of the relationship between dietary GI and GL and measures of body fatness.

At present, the only way to obtain unbiased information on energy requirements in free-living settings is to use doubly labelled water as a biomarker ${ }^{(25)}$. This technique is expensive and impractical for application to large-scale epidemiological studies. Instead, we calculated EER with the use of equations from the US Dietary Reference Intakes ${ }^{(43)}$. In the absence of measured TEE, these equations with high $R^{2}$ values ( 0.82 for men and 0.79 for women) ${ }^{(43)}$ should serve as the best proxy, although the selection of physical activity category was based on self-report (i.e. $7 \mathrm{~d}$ physical activity diary), which may be susceptible to reporting bias. Additionally, we do not know the sensitivity and specificity of the procedure for identifying EI misreporters used. If misclassification between AR and UR is big, the real differences in the associations of GI and GL with food and nutrient intake and obesity would be attenuated to be not significant. Nonetheless, we are confident of our conclusions, because the associations observed in the entire populations were similarly observed in AR.

Another limitation of the present study is the relatively low response rate (61\%), and only $40 \%$ of the eligible sample was included in the present study. The subjects included in the present analysis ( $n$ 1487) differed somewhat from those excluded from the analysis ( $n$ 705-758 depending on the variables). The excluded subjects were more likely to be younger, be in manual occupations and be current smokers 
(all $P<0 \cdot 05$ ). However, a previous analysis has concluded that there is no evidence to suggest serious non-response bias in the NDNS ${ }^{(35)}$. Although GI values were carefully determined with, for example, consideration of cooking methods (e.g. boiled, fried, mashed and canned potatoes) as much as possible, general limitations to assigning appropriate GI values should be acknowledged, including the still restricted number of items in the GI table, the inclusion of mainly American or Australian food items, the lack of values of mixed dishes and the lack of information on differences in variety (e.g. potato and rice), degree of ripeness (e.g. banana), composition (e.g. more or less fat), cooking methods and product formulations of the same brand ${ }^{(40,52)}$. Finally, although we adjusted for a variety of potential confounding variables, residual confounding could not be ruled out.

In conclusion, in the present cross-sectional study in British adults, a high-GI diet, which was high in breads and potatoes but low in fruit, other cereals and dairy products, showed an independent positive association with the risk of general and central obesity in both men and women. Dietary GL, which was strongly correlated with carbohydrate intake, showed an independent positive association with the risk of central obesity in both sexes and general obesity in only women. These associations were similarly observed in the subjects with a plausible EI. Conversely, in the subjects with an implausibly low EI, the associations were generally weaker and many of them failed to reach statistical significance. Thus, underreporting of EI seemed to confound the positive associations of dietary GI and GL with general and central obesity by reducing them. Further research (particularly with a prospective design) in diverse populations is needed, taking into account dietary misreporting as well as using different dietary assessment methods so that any firm conclusions can be drawn with regard to the effect of dietary GI and GL on excess body fatness. More generally, as misreporting of dietary intake is a well-known phenomenon in nutritional epidemiology ${ }^{(25-32)}$ and there is not enough knowledge of differential misreporting among foods and nutrients ${ }^{(28-31)}$, the routine application of some procedures to identify and separately treat those who report data of poor validity ${ }^{(33,44,49)}$ would improve the precision and accuracy of results in studies of diet and health.

\section{Acknowledgements}

The present study was supported in part by the JSPS Postdoctoral Fellowships for Research Abroad, Japan Society for the Promotion of Science, Japan. K. M. designed the study, analysed and interpreted the data, and wrote the manuscript. T. A. M. and M. B. E. L. designed the study and helped to write the manuscript. All authors read and approved the final manuscript. None of the authors has a conflict of interest.

\section{References}

1. Ludwig DS (2002) The glycemic index: physiological mechanisms relating to obesity, diabetes, and cardiovascular disease. JAMA 287, 2414-2423.
2. Stevenson EJ, Williams C, Mash LE, et al. (2006) Influence of high-carbohydrate mixed meals with different glycemic indexes on substrate utilization during subsequent exercise in women. Am J Clin Nutr 84, 354-360.

3. Pereira MA, Swain J, Goldfine AB, et al. (2004) Effects of a low-glycemic load diet on resting energy expenditure and heart disease risk factors during weight loss. JAMA 292, 2482-2490.

4. Augustin LS, Franceschi S, Jenkins DJ, et al. (2002) Glycemic index in chronic disease: a review. Eur J Clin Nutr 56, 1049-1071.

5. Lau C, Toft U, Tetens I, et al. (2006) Association between dietary glycemic index, glycemic load, and body mass index in the Inter99 study: is underreporting a problem? Am J Clin Nutr 84, 641-645.

6. Murakami K, Sasaki S, Okubo H, et al. (2007) Dietary fiber intake, dietary glycemic index and load, and body mass index: a cross-sectional study of 3931 Japanese women aged 18-20 years. Eur J Clin Nutr 61, 986-995.

7. Murakami K, Sasaki S, Takahashi Y, et al. (2006) Dietary glycemic index and load in relation to metabolic risk factors in Japanese female farmers with traditional dietary habits. $A m J$ Clin Nutr 83, 1161-1169.

8. Youn S, Woo HD, Cho YA, et al. (2012) Association between dietary carbohydrate, glycemic index, glycemic load, and the prevalence of obesity in Korean men and women. Nutr Res 32, 153-159.

9. Finley CE, Barlow CE, Halton TL, et al. (2010) Glycemic index, glycemic load, and prevalence of the metabolic syndrome in the Cooper Center Longitudinal Study. J Am Diet Assoc 110, 1820-1829.

10. Ma Y, Olendzki B, Chiriboga D, et al. (2005) Association between dietary carbohydrates and body weight. Am J Epidemiol 161, 359-367.

11. Du H, van der ADL, van Bakel MME, et al. (2009) Dietary glycaemic index, glycaemic load and subsequent changes of weight and waist circumference in European men and women. Int J Obes 33, 1280-1288.

12. Hare-Bruun H, Flint A \& Heitmann BL (2006) Glycemic index and glycemic load in relation to changes in body weight, body fat distribution, and body composition in adult Danes. Am J Clin Nutr 84, 871-879.

13. Du H, van der ADL, van Bakel MME, et al. (2008) Glycemic index and glycemic load in relation to food and nutrient intake and metabolic risk factors in a Dutch population. Am J Clin Nutr 87, 655-661.

14. Mendez MA, Covas MI, Marrugat J, et al. (2009) Glycemic load, glycemic index, and body mass index in Spanish adults. Am J Clin Nutr 89, 316-322.

15. Milton JE, Briche B, Brown IJ, et al. (2007) Relationship of glycaemic index with cardiovascular risk factors: analysis of the National Diet and Nutrition Survey for people aged 65 and older. Public Health Nutr 10, 1321-1335.

16. Liese AD, Schulz M, Fang F, et al. (2005) Dietary glycemic index and glycemic load, carbohydrate and fiber intake, and measures of insulin sensitivity, secretion, and adiposity in the Insulin Resistance Atherosclerosis study. Diabetes Care 28, 2832-2838.

17. Rossi M, Bosetti C, Talamini R, et al. (2010) Glycemic index and glycemic load in relation to body mass index and waist to hip ratio. Eur J Nutr 49, 459-464.

18. Sahyoun NR, Anderson AL, Kanaya AM, et al. (2005) Dietary glycemic index and load, measures of glucose metabolism, and body fat distribution in older adults. Am J Clin Nutr 82, $547-552$. 
19. Schulz M, Liese AD, Mayer-Davis EJ, et al. (2005) Nutritional correlates of dietary glycaemic index: new aspects from a population perspective. Br J Nutr 94, 397-406.

20. van Bakel MM, Kaaks R, Feskens EJ, et al. (2009) Dietary glycaemic index and glycaemic load in the European Prospective Investigation into Cancer and Nutrition. Eur J Clin Nutr 63, Suppl. 4, S188-S205.

21. Mayer-Davis EJ, Dhawan A, Liese AD, et al. (2006) Towards understanding of glycaemic index and glycaemic load in habitual diet: associations with measures of glycaemia in the Insulin Resistance Atherosclerosis Study. Br J Nutr 95, 397-405.

22. Foster-Powell K, Holt SH \& Brand-Miller JC (2002) International table of glycemic index and glycemic load values: 2002. Am J Clin Nutr 76, 5-56.

23. Atkinson FS, Foster-Powell K \& Brand-Miller JC (2008) International tables of glycemic index and glycemic load values: 2008. Diabetes Care 31, 2281-2283.

24. Louie JCY, Flood V, Turner N, et al. (2010) Methodology for adding glycemic index values to 24-hour recalls. Nutrition 27, 59-64.

25. Livingstone MBE \& Black AE (2003) Markers of the validity of reported energy intake. J Nutr 133, Suppl. 3, 895S-920S

26. Mattisson I, Wirfalt E, Aronsson CA, et al. (2005) Misreporting of energy: prevalence, characteristics of misreporters and influence on observed risk estimates in the Malmo Diet and Cancer cohort. Br J Nutr 94, 832-842.

27. Lutomski JE, van den Broeck J, Harrington J, et al. (2011) Sociodemographic, lifestyle, mental health and dietary factors associated with direction of misreporting of energy intake. Public Health Nutr 14, 532-541.

28. Subar AF, Kipnis V, Troiano RP, et al. (2003) Using intake biomarkers to evaluate the extent of dietary misreporting in a large sample of adults: the OPEN study. Am J Epidemiol 158, $1-13$.

29. Rosell MS, Hellenius MLB, De Faire UH, et al. (2003) Associations between diet and the metabolic syndrome vary with the validity of dietary intake data. Am J Clin Nutr 78, 84-90.

30. Heitmann BL \& Lissner L (1995) Dietary underreporting by obese individuals: is it specific or non-specific? BMJ 311, 986-989.

31. Murakami K, Sasaki S, Takahashi Y, et al. (2008) Misreporting of dietary energy, protein, potassium and sodium in relation to body mass index in young Japanese women. Eur J Clin Nutr 62, 111-118.

32. Nielsen BM, Nielsen MM, Toubro S, et al. (2009) Past and current body size affect validity of reported energy intake among middle-aged Danish men. J Nutr 139, 2337-2343.

33. Black AE (2000) The sensitivity and specificity of the Goldberg cut-off for EI:BMR for identifying diet reports of poor validity. Eur J Clin Nutr 54, 395-404.

34. Tooze JA, Krebs-Smith SM, Troiano RP, et al. (2012) The accuracy of the Goldberg method for classifying misreporters of energy intake on a food frequency questionnaire and 24-h recalls: comparison with doubly labeled water. Eur J Clin Nutr 66, 569-576.

35. Food Standards Agency (2010) NDNS previous survey reports. http://webarchive.nationalarchives.gov.uk/2010040 6130654/food.gov.uk/science/dietarysurveys/ndnsdocuments/ ndnsprevioussurveyreports/

36. World Health Organization (2000) Obesity: Preventing and Managing the Global Epidemic. Report of a WHO Consultation. WHO Technical Report Series no. 894. Geneva: WHO.
37. Ashwell M, Gunn P \& Gibson S (2012) Waist-to-height ratio is a better screening tool than waist circumference and BMI for adult cardiometabolic risk factors: systematic review and meta-analysis. Obes Rev 13, 275-286.

38. Smithers G (1993) MAFF's nutrient databank. Nutr Food Sci 93, 16-19.

39. Food Standards Agency (2002) McCance and Widdowson's The Composition of Foods, 6th ed. Cambridge: Royal Society of Chemistry.

40. van Bakel MM, Slimani N, Feskens EJ, et al. (2009) Methodological challenges in the application of the glycemic index in epidemiological studies using data from the European Prospective Investigation into Cancer and Nutrition. $J$ Nutr 139, 568-575.

41. Flood A, Subar AF, Hull SG, et al. (2006) Methodology for adding glycemic load values to the National Cancer Institute Diet History Questionnaire database. J Am Diet Assoc 106, 393-402.

42. Ainsworth BE, Haskell WL, Herrmann SD, et al. (2011) 2011 Compendium of Physical Activities: a second update of codes and MET values. Med Sci Sports Exerc 43, 1575-1581.

43. Institute of Medicine (2002) Dietary Reference Intakes for Energy, Carbohydrate, Fiber, Fat, Fatty Acids, Cholesterol, Protein and Amino Acids. Washington, DC: National Academy Press.

44. Huang TT, Roberts SB, Howarth NC, et al. (2005) Effect of screening out implausible energy intake reports on relationships between diet and BMI. Obes Res 13, 1205-1217.

45. Black AE \& Cole TJ (2000) Within- and between-subject variation in energy expenditure measured by the doublylabelled water technique: implications for validating reported dietary energy intake. Eur J Clin Nutr 54, 386-394.

46. Willett WC, Howe GR \& Kushi LH (1997) Adjustment for total energy intake in epidemiologic studies. Am J Clin Nutr $\mathbf{6 5}$, Suppl. 4, 1220S-1228S.

47. Okubo H, Sasaki S, Hirota N, et al. (2006) The influence of age and body mass index to relative accuracy of energy intake among Japanese adults. Public Health Nutr 9, 651-657.

48. Barnard JA, Tapsell LC, Davies PSW, et al. (2002) Relationship of high energy expenditure and variation in dietary intake with reporting accuracy on 7 day food records and diet histories in a group of healthy adult volunteers. Eur $J$ Clin Nutr 56, 358-367.

49. Mendez MA, Popkin BM, Buckland G, et al. (2011) Alternative methods of accounting for underreporting and overreporting when measuring dietary intake-obesity relations. Am J Epidemiol 173, 448-458.

50. Bingham S, Luben R, Welch A, et al. (2008) Associations between dietary methods and biomarkers, and between fruits and vegetables and risk of ischaemic heart disease, in the EPIC Norfolk Cohort Study. Int J Epidemiol 37, 978-987.

51. George SM, Thompson FE, Midthune D, et al. (2012) Strength of the relationships between three self-reported dietary intake instruments and serum carotenoids: the Observing Energy and Protein Nutrition (OPEN) Study. Public Health Nutr 15, 1000-1007.

52. Henry CJK, Lightowler HJ, Strik CM, et al. (2005) Glycaemic index values for commercially available potatoes in Great Britain. Br J Nutr 94, 917-921. 